\title{
The Prognostic Value of C-reactive Protein to Albumin Ratio in Patients with Isolated Degenerative Aortic Valve Stenosis Undergoing Surgical Aortic Valve Replacement
}

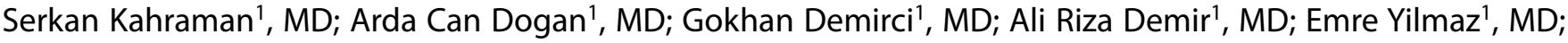

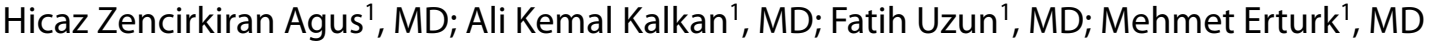

DOI: 10.21470/1678-9741-2019-0114

\begin{abstract}
Objective: To evaluate the prognostic value of C-reactive protein to albumin ratio (CAR) in patients with severe aortic valve stenosis undergoing surgical aortic valve replacement (AVR).

Methods: Four hundred seventy-six patients with severe degenerative aortic stenosis who underwent successful isolated surgical AVR were enrolled. Hospitalization due to heart failure, surgical aortic reoperation, paravalvular leakage rates, and longterm mortality were evaluated in the whole study group. The participants were divided into two groups, as 443 patients without mortality (group 1) and 33 patients with mortality (group 2) during the follow-up time.

Results: CAR was lower in patients without mortality than in those with mortality during the follow-up time $(0.84$ [0.03-23.43] vs. 2.50 [0.22-26.55], respectively, $P<0.001$ ). Age (odds ratio [OR]: 1.062, confidence interval $[\mathrm{CI}]: 1.012-1.114, P=0.014)$, CAR (OR
\end{abstract}

1.221, Cl: 1.125-1.325, $P<0.001$ ), ejection fraction (OR: $0.956, \mathrm{Cl}:$ 0.916-0.998, $P=0.042$ ), and valve type (OR: $2.634, \mathrm{Cl}: 1.045-6.638$, $P=0.040$ ) were also found to be independent predictors of long-term mortality. Additionally, rehospitalization (0.86 [0.03-26.55] vs. 1.6 [0.17-24.05], $P=0.006)$, aortic reoperation (0.87 [0.03-26.55] vs. 1.6 [0.20-23.43], $P=0.016)$, and moderate to severe aortic paravalvular leakage (0.86 [0.03-26.55] vs. 1.86 [0.21-19.50], $P=0.023)$ ratios were associated with higher CAR.

Conclusion: It was firstly described that CAR was strongly related with increased mortality rates in patients with isolated severe aortic stenosis after surgical AVR. Additionally, rehospitalization, risk of paravalvular leakage, and aortic reoperation rates were higher in patients with increased CAR than in those without it.

Keywords: Albumin. Aortic Valve Stenosis. C-Reactive Protein. Heart Valve Prosthesis. Hospitalization. Reoperation.

\begin{tabular}{ll}
\hline \multicolumn{2}{l}{ Abbreviations, acronyms \& symbols } \\
\hline ACEI & $=$ Angiotensin-converting enzyme inhibitor \\
ARB & $=$ Angiotensin receptor blocker \\
AVR & $=$ Aortic valve replacement \\
CAR & $=$ C-reactive protein to albumin ratio \\
CI & $=$ Confidence interval \\
COPD & $=$ Chronic obstructive pulmonary disease \\
CRP & $=$ C-reactive protein \\
EF & $=$ Ejection fraction \\
HDL & $=$ High-density lipoprotein
\end{tabular}

'Department of Cardiology, University of Health Sciences, Istanbul Mehmet Akif Ersoy Thoracic and Cardiovascular Surgery Center, Training and Research Hospital, Istanbul, Turkey.

This study was carried out at the Department of Cardiology, University of Health Sciences, Istanbul Mehmet Akif Ersoy Thoracic and Cardiovascular Surgery Center, Training and Research Hospital, Istanbul, Turkey.
IL-6
= Interleukin-6
LDL = Low-density lipoprotein
LVEDD = Left ventricular end-diastolic diameter
LVESD = Left ventricular end-systolic diameter
NYHA $=$ New York Heart Association
OR = Odds ratio
SPSS = Statistical Package for Social Sciences
TG = Triglyceride

Correspondence Address:

Serkan Kahraman

(iD) https://orcid.org/0000-0003-2796-0987

Department of Cardiology, University of Health Sciences, Istanbul Mehmet Akif Ersoy Thoracic and Cardiovascular Surgery Center, Training and Research Hospital İstasyon Mah. Turgut Özal Bulvarı No:11 Küçükçekmece, Istanbul, Turkey

Zip Code: 34114

E-mail: serkankahraman_86@outlook.com 


\section{INTRODUCTION}

Aortic stenosis is the most common form of degenerative valvular heart disease, with increasing prevalence, and it is still the leading cause of surgical valve replacement therapy, especially in developing countries ${ }^{[1]}$. Although several etiological risk factors of aortic stenosis have been described, it is known that degenerative valvular stenosis is an active process with inflammatory histological valve changes, like atherosclerotic plaques $^{[2]}$. Thus, the chronic inflammatory process plays an important role on atherosclerosis, an uncontested underlying pathogenic mechanism of degenerative valvular aortic stenosis.

High-sensitive C-reactive protein (CRP) is one of the most specific biomarkers of systemic inflammation. It was demonstrated that serum CRP levels predict progression and severity of aortic stenosis due to the pathogenic role of inflammation on valvular disease ${ }^{[3]}$. Contrary to CRP, albumin is reduced in the chronic inflammatory process, as a negative acute phase responder ${ }^{[4]}$. Accordingly, CRP to albumin ratio (CAR) is a more sensitive marker to predict inflammation than CRP or albumin rates alone, due to its two different directions (increased CRP and decreased albumin). While inflammatory markers have been evaluated on severity and prognosis of aortic stenosis, they have not been adequately studied to determine their effect on the prognosis after aortic valve replacement (AVR) therapy. We aimed to evaluate the prognostic value of CAR on clinical events in patients with isolated severe degenerative aortic stenosis undergoing surgical AVR.

\section{METHODS}

\section{Study Population}

This retrospective observational study was conducted at a tertiary high-volume center in Turkey from January 2010 to December 2017. Eight hundred seventy-six patients with severe degenerative aortic stenosis who underwent successful surgical AVR were observed. After exclusion, four hundred seventy-six (476) patients were enrolled in this study. Patients undergoing urgent surgery due to acute aortic regurgitation and patients with active infection, with known coronary artery disease, undergoing percutaneous coronary intervention, with previously or simultaneously coronary artery bypass grafting or cardiac valve replacement surgery other than aortic valve, with type A aortic dissection, malignancy, or end-stage hepatic or renal disease were excluded from the study. Patients with aortic mismatch after operation were also excluded from the study. The participants were divided into two groups, as patients without mortality (group 1) and with mortality (group 2) during the follow-up time. The study was approved by the local ethical committee.

\section{Biochemical Analysis}

All biochemical analyses were measured before performing AVR. Total cholesterol, low-density lipoprotein (LDL) cholesterol, high-density lipoprotein (HDL) cholesterol, triglyceride (TG), fasting blood glucose, blood urea nitrogen, creatinine, and complete blood count were measured after stopping the oral intake for eight to 12 hours and blood samples were drawn from the brachial veins. CRP and serum albumin levels were measured by using The Roche Diagnostics Cobas ${ }^{\circledR} 8000$ c502 analyzer (Indianapolis, United States of America). The value of 0.0-5.0 mg/l was accepted as normal range for CRP and the value of 3.5-5.2 g/ dl was accepted for serum albumin.

\section{Clinical Follow-up}

To evaluate the effect of low and high levels of CAR in patients with severe aortic stenosis undergoing surgical AVR on long-term clinical events, including hospitalization due to heart failure, surgical aortic reoperation and all-cause death and mortality were compared. Patients' follow-up visits were done at hospital admission for suitable patients, and telephone followup visits were done for others.

\section{Statistical Analysis}

Statistical analysis was made using the Statistical Package for Social Sciences (IBM SPSS Statistics for Windows, version 21.0, released in 2012, IBM Corp., Armonk, New York, United States of America) computer software. Data were expressed as "n (\%)" for categorical variables; Pearson's chi-square and Fisher's exact tests were performed for categorical variables. After fitness to normal distribution was analyzed with the Kolmogorov-Smirnov test, data were expressed as "median (minimum-maximum)" for abnormal distribution. Mann-Whitney $U$ test was used for comparing quantitative variables with abnormal distribution. Univariate and multivariate logistic regression analyses were used to determine the independent predictors of mortality after surgical AVR. A P-value $<0.05$ was considered statistically significant.

\section{RESULTS}

Basal demographic and clinical variables of the whole study group were demonstrated in Table 1. Patients without mortality during the follow-up time formed group 1 and patients with mortality formed group 2. There were no significant differences in gender; smoking status; diabetes mellitus; hypertension; dyslipidemia; peripheral arterial disease; atrial fibrillation presence; chronic obstructive pulmonary disease; previous cerebrovascular disease; history of congestive heart failure; symptoms such as dyspnea, angina, and syncope; levels of leukocyte, thrombocyte, total cholesterol, LDL cholesterol, HDL cholesterol, TG, and albumin; medication usage (beta-blocker, calcium channel blocker, and statin); aortic valve area; maximum and mean aortic gradients; left ventricular end-diastolic and -systolic diameters; and postoperative hospital discharging time between the groups.

Patients without mortality (group 1) were younger than patients with mortality (group 2) (61 [40-88] years vs. 74 [46-86] years, respectively, $P<0.001)$. While creatinine (0.86 [0.40-8.60] vs. $1.0[0.50-4.60], P=0.003)$ and CRP (3.5 [0.3-82.0] vs. 10.0 [1.093.8], $P<0.001)$ levels in group 1 were lower than in group 2 , hemoglobin level was higher in group 1 than in group 2 (13.2 [6.6-18.0] vs. 11.9 [8.1-16.5], $P=0.011$ ). Angiotensin-converting enzyme inhibitor (ACEI) or angiotensin receptor blocker (ARB) 
Table 1. Basal demographic and clinical variables of both groups with mortality absence and presence during the follow-up time.

\begin{tabular}{|c|c|c|c|c|}
\hline & & $\begin{array}{l}\text { Mortality absence } \\
\text { Group } 1(n=443)\end{array}$ & $\begin{array}{l}\text { Mortality presence } \\
\text { Group } 2(n=33)\end{array}$ & $P$-value \\
\hline \multicolumn{2}{|l|}{ Age (years) } & $61(40-88)$ & $74(46-86)$ & $<0.001$ \\
\hline \multicolumn{2}{|l|}{ Gender (female) \% (n) } & $31.4(139)$ & $45.5(15)$ & 0.095 \\
\hline \multicolumn{2}{|l|}{ Smoking \% (n) } & $25.1(111)$ & $21.2(7)$ & 0.622 \\
\hline \multicolumn{2}{|l|}{ Diabetes mellitus \% (n) } & $23.9(106)$ & $24.2(8)$ & 0.967 \\
\hline \multicolumn{2}{|l|}{ Hypertension \% (n) } & $49.7(220)$ & $39.4(13)$ & 0.255 \\
\hline \multicolumn{2}{|l|}{ Dyslipidemia \% (n) } & $28.4(126)$ & $27.3(9)$ & 0.886 \\
\hline \multicolumn{2}{|c|}{ Peripheral arterial disease \% (n) } & $2.9(13)$ & $6.1(2)$ & 0.279 \\
\hline \multicolumn{2}{|l|}{ Atrial fibrillation \% (n) } & $8.4(37)$ & $12.1(4)$ & 0.314 \\
\hline \multicolumn{2}{|l|}{ COPD \% (n) } & $14.7(65)$ & $9.1(3)$ & 0.277 \\
\hline \multicolumn{2}{|c|}{ Previous cerebrovascular disease \% (n) } & $0.2(1)$ & $3(1)$ & 0.134 \\
\hline \multicolumn{2}{|c|}{ Previous congestive heart failure $\%(n)$} & $14.9(66)$ & $27.3(9)$ & 0.060 \\
\hline \multirow{2}{*}{ Dyspnea \% (n) } & NYHA I-II & $44.2(196)$ & $27.3(9)$ & 0.058 \\
\hline & NYHA III-IV & $55.8(247)$ & $72.7(24)$ & \\
\hline \multicolumn{2}{|l|}{ Angina \% (n) } & $50.3(223)$ & $60.6(20)$ & 0.255 \\
\hline \multicolumn{2}{|l|}{ Syncope \% (n) } & $9.9(44)$ & $15.2(5)$ & 0.244 \\
\hline \multicolumn{2}{|l|}{ Creatinine (mg/dl) } & $0.86(0.40-8.60)$ & $1.0(0.50-4.60)$ & 0.003 \\
\hline \multicolumn{2}{|l|}{ Hemoglobin (g/dl) } & $13.2(6.6-18.0)$ & $11.9(8.1-16.5)$ & 0.011 \\
\hline \multicolumn{2}{|l|}{ Leukocytes $\times 10^{3} / \mathrm{mm}^{3}$} & $7.9(2.1-24.5)$ & $8.1(4.0-27.7)$ & 0.699 \\
\hline \multicolumn{2}{|l|}{ Thrombocyte $\times 10^{3} / \mathrm{mm}^{3}$} & $234(37-565)$ & $231(101-380)$ & 0.376 \\
\hline \multicolumn{2}{|l|}{ Total cholesterol (mg/dl) } & $178(67-330)$ & $172(118-282)$ & 0.859 \\
\hline \multicolumn{2}{|l|}{ LDL cholesterol (mg/dl) } & $106(10-225)$ & $101(63-188)$ & 0.824 \\
\hline \multicolumn{2}{|l|}{ HDL cholesterol (mg/dl) } & $42(9-111)$ & $48(17-110)$ & 0.324 \\
\hline \multicolumn{2}{|l|}{ Triglyceride (mg/dl) } & $121(16-613)$ & $140(53-800)$ & 0.496 \\
\hline \multicolumn{2}{|l|}{$\mathrm{CRP}(\mathrm{mg} / \mathrm{l})$} & $3.5(0.3-82.0)$ & $10.0(1.0-93.8)$ & $<0.001$ \\
\hline \multicolumn{2}{|l|}{ Albumin (g/dl) } & $4.2(2.1-37.6)$ & $4.2(1.7-4.9)$ & 0.445 \\
\hline \multicolumn{2}{|l|}{ CRP to albumin ratio } & $0.84(0.03-23.43)$ & $2.50(0.22-26.55)$ & $<0.001$ \\
\hline \multirow{4}{*}{ Medication usage $\%$ (n) } & Beta-blocker & $80.8(358)$ & $66.7(22)$ & 0.051 \\
\hline & ACEl or ARB & $43.8(194)$ & $18.2(6)$ & 0.004 \\
\hline & Calcium channel blocker & $13.3(59)$ & $12.1(4)$ & 0.551 \\
\hline & Statin & $22.1(98)$ & $18.2(6)$ & 0.597 \\
\hline Ejection fraction (\%) & & $60(15-70)$ & $55(25-65)$ & 0.001 \\
\hline Aortic valve area $\left(\mathrm{cm}^{2}\right)$ & & $0.7(0.3-0.9)$ & $0.7(0.4-0.9)$ & 0.234 \\
\hline Maximum gradient ( $\mathrm{mm}$ & & $76(40-181)$ & $74(43-154)$ & 0.667 \\
\hline Mean gradient $(\mathrm{mmHg})$ & & $50(25-119)$ & $46(26-90)$ & 0.220 \\
\hline $\operatorname{LVEDD~}(\mathrm{mm})$ & & $50(33-85)$ & $50(38-68)$ & 0.525 \\
\hline $\operatorname{LVESD}(\mathrm{mm})$ & & $32(19-78)$ & $33(20-59)$ & 0.953 \\
\hline Left atrial diameter $(\mathrm{mm}$ & & $39(22-59)$ & $42(28-52)$ & 0.012 \\
\hline 10 lo tung o ( & Bioprosthetic valve & $29.1(129)$ & $57.6(19)$ & 0.001 \\
\hline vaive sype to (II) & Mechanical prosthetic valve & $70.9(314)$ & $42.4(14)$ & \\
\hline Postoperative dischargil & g time (days) & $9(5-80)$ & $9(5-64)$ & 0.517 \\
\hline Follow-up time (month & & $51(2-300)$ & $72(1-267)$ & $<0.001$ \\
\hline
\end{tabular}

$\mathrm{ACEl}=$ angiotensin-converting enzyme inhibitor; $\mathrm{ARB}=$ angiotensin receptor blocker; $\mathrm{COPD}=$ chronic obstructive pulmonary disease; $\mathrm{CRP}=\mathrm{C}$-reactive protein; $\mathrm{HDL}=$ high-density lipoprotein; $\mathrm{LDL}=$ low-density lipoprotein; $\mathrm{LVEDD=left}$ ventricular end-diastolic diameter; $\mathrm{LVESD}=$ left ventricular end-systolic diameter; NYHA=New York Heart Association 
usage rate was higher in group 1 than in group 2 (43.8\% [194] vs. $18.2 \%[6], P=0.004)$. Ejection fraction (EF) was also higher in group 1 than in group 2 (60 [15-70] vs. 55 [25-65], $P=0.001)$. However, left atrial diameter was narrower in group 1 than in group 2 (39 [22-59] vs. 42 [28-52], $P=0.012)$. Mechanical prosthetic valve ratio was higher in group 1 than in group 2 (70.9\% [314] vs. 42.4\% [14], $P=0.001)$. And CAR was lower in group 1 than in group $2(0.84$ [0.03-23.43] vs. 2.50 [0.22-26.55], $P<0.001$ ) (Figure 1). Additionally, follow-up time after surgical AVR was shorter in group 1 than in group 2 (51 [2-300] months vs. 72 [1-267] months, $P<0.001$ ).
In multivariate logistic regression analysis, age (odds ratio [OR]: 1.062, confidence interval [CI]: 1.012-1.114, $P=0.014), C A R$ (OR: 1.221, Cl: 1.125-1.325, $P<0.001$ ), EF (OR: 0.956, Cl: 0.916-0.998, $P=0.042$ ), and valve type (OR: 2.634, $C l: 1.045-6.638, P=0.040$ ) were found to be independent predictors of long-term mortality in patients with severe aortic stenosis undergoing surgical AVR (Table 2).

Additionally, rehospitalization $(0.86[0.03-26.55]$ vs. 1.6 [0.1724.05], $P=0.006)$, aortic reoperation (0.87 [0.03-26.55] vs. $1.6[0.20-$ 23.43], $P=0.016)$, and moderate to severe aortic paravalvular

Table 2. Univariate and multivariate logistic regression analyses giving information about independent predictors of mortality.

\begin{tabular}{|c|c|c|c|c|c|c|}
\hline & \multicolumn{3}{|c|}{ Univariate analysis } & \multicolumn{3}{|c|}{ Multivariate analysis } \\
\hline & Odds ratio & $\begin{array}{c}95 \% \mathrm{Cl} \\
\text { (lower-upper) }\end{array}$ & $P$-value & Odds ratio & $\begin{array}{c}95 \% \mathrm{Cl} \\
\text { (lower-upper) }\end{array}$ & $P$-value \\
\hline Age & 1.075 & $1.038-1.113$ & $<0.001$ & 1.062 & $1.012-1.114$ & 0.014 \\
\hline Gender (female) & 0.549 & $0.269-1.120$ & 0.099 & & & \\
\hline Smoking & 1.242 & $0.525-2.940$ & 0.622 & & & \\
\hline Diabetes mellitus & 0.983 & $0.431-2.244$ & 0.967 & & & \\
\hline Hypertension & 1.518 & $0.737-3.126$ & 0.258 & & & \\
\hline Dyslipidemia & 1.060 & $0.479-2.343$ & 0.886 & & & \\
\hline Peripheral arterial disease & 0.469 & $0.101-2.170$ & 0.332 & & & \\
\hline Atrial fibrillation & 0.661 & $0.220-1.981$ & 0.460 & & & \\
\hline COPD & 1.720 & 0.510-5.799 & 0.382 & & & \\
\hline Previous cerebrovascular disease & 0.072 & $0.004-1.185$ & 0.066 & & & \\
\hline Previous congestive heart failure & 0.467 & 0.208-1.049 & 0.065 & & & \\
\hline Dyspnea & 2.116 & $0.962-4.656$ & 0.063 & & & \\
\hline Angina & 0.659 & $0.320-1.357$ & 0.258 & & & \\
\hline Syncope & 0.618 & $0.227-1.681$ & 0.345 & & & \\
\hline Creatinine & 1.544 & $1.044-2.283$ & 0.029 & 1.212 & $0.747-1.966$ & 0.436 \\
\hline Hemoglobin & 0.811 & $0.690-0.953$ & 0.011 & 1.089 & $0.883-1.342$ & 0.427 \\
\hline Leukocytes & 1.032 & $0.912-1.167$ & 0.621 & & & \\
\hline Thrombocyte & 0.997 & 0.992-1.002 & 0.268 & & & \\
\hline Total cholesterol & 1.001 & 0.993-1.008 & 0.845 & & & \\
\hline LDL cholesterol & 0.999 & $0.989-1.008$ & 0.779 & & & \\
\hline HDL cholesterol & 1.015 & 0.990-1.040 & 0.236 & & & \\
\hline Triglyceride & 1.003 & 0.999-1.006 & 0.151 & & & \\
\hline CRP to albumin ratio & 1.242 & $1.157-1.334$ & $<0.001$ & 1.221 & $1.125-1.325$ & $<0.001$ \\
\hline Ejection fraction & 0.955 & $0.926-0.984$ & 0.003 & 0.956 & $0.916-0.998$ & 0.042 \\
\hline LVEDD & 0.984 & $0.941-1.030$ & 0.490 & & & \\
\hline LVESD & 1.008 & 0.971-1.047 & 0.675 & & & \\
\hline Left atrial diameter & 1.066 & $1.007-1.129$ & 0.029 & 0.977 & 0.903-1.057 & 0.558 \\
\hline Postoperative discharging time & 1.039 & $1.012-1.067$ & 0.005 & 1.033 & 0.998-1.069 & 0.067 \\
\hline Valve type & 3.303 & $1.608-6.788$ & 0.001 & 2.634 & $1.045-6.638$ & 0.040 \\
\hline
\end{tabular}

$\mathrm{Cl}=$ confidence interval; $\mathrm{COPD}=$ chronic obstructive pulmonary disease; $\mathrm{CRP}=\mathrm{C}$-reactive protein; $\mathrm{HDL}=$ high-density lipoprotein; LDL=low-density lipoprotein; LVEDD=left ventricular end-diastolic diameter; LVESD=left ventricular end-systolic diameter 
leakage (0.86 [0.03-26.55] vs. 1.86 [0.21-19.50], $P=0.023)$ ratios were associated with higher CAR (Table 3 and Figure 1).

\section{DISCUSSION}

In our retrospective study, to the best of our knowledge, it was firstly demonstrated that higher CAR was found to be strongly associated with increased mortality rates in patients with severe degenerative aortic stenosis undergoing surgical AVR. Additionally, hospitalization rates due to heart failure after AVR were related with higher CAR. Surgical aortic reoperation and moderate to high paravalvular leakage ratios were also associated with higher CAR.

Table 3. Comparison of CRP to albumin ratio in the presence and absence of rehospitalization, aortic reoperation, and moderate to severe paravalvular leak.

\begin{tabular}{l|c|c|c}
\hline & Rehospitalization absence & Rehospitalization presence & $\boldsymbol{P}$-value \\
\hline CRP to albumin ratio & $0.86(0.03-26.55)$ & $1.6(0.17-24.05)$ & 0.006 \\
\hline & Aortic reoperation absence & Aortic reoperation presence & $\boldsymbol{P}$-value \\
\hline CRP to albumin ratio & $0.87(0.03-26.55)$ & $1.6(0.20-23.43)$ & 0.016 \\
\hline CRP to albumin ratio & None to mild paravalvular leak & Moderate to severe paravalvular leak & $\boldsymbol{P}$-value \\
\hline
\end{tabular}

$\mathrm{CRP}=\mathrm{C}$-reactive protein
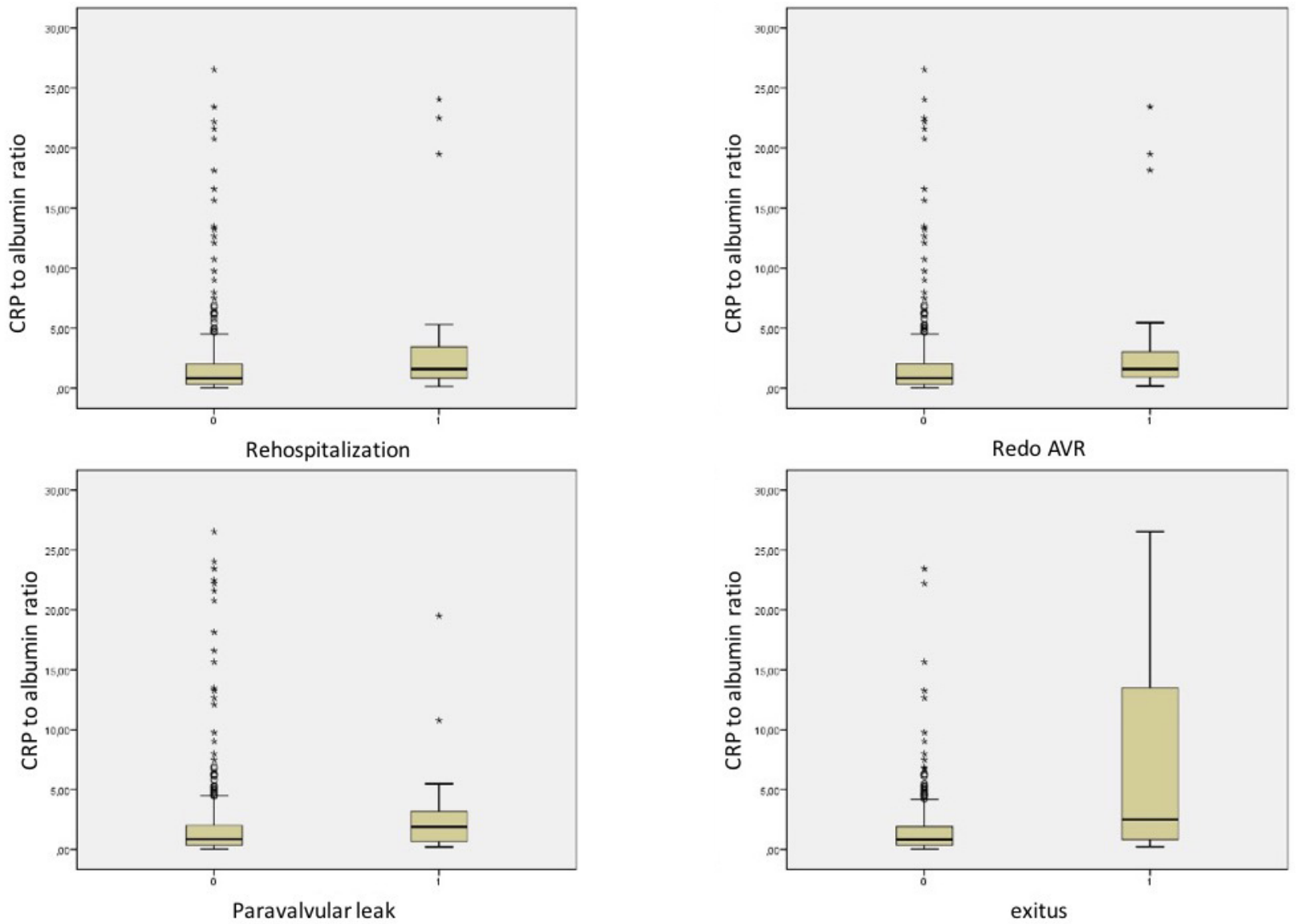

Fig. 1 - Comparison of serum CRP to albumin ratio in the whole study group according to rehospitalization, aortic reoperation, paravalvular leakage, and mortality rates.

AVR=aortic valve replacement; $C R P=C$-reactive protein 
Inflammation is known as an important risk factor for cardiovascular diseases ${ }^{[5]}$. Histopathological findings of inflammation including calcification, fibrosis, and lipid storage have been demonstrated in degenerative valvular aortic stenosis, like atherosclerosis ${ }^{[2]}$. Due to the mentioned reason, several biochemical markers have been described to detect inflammation on progression and prognosis of aortic stenosis. CRP was firstly described by Tillet and Francis as a marker of ongoing inflammation. In the event of chronic inflammatory process, CRP, which is produced in the liver by interleukin-6 (IL-6), tumor necrosis factor $a$, and other pro-inflammatory cytokines, has also been localized in the aortic valve tissue, as well as T lymphocytes and macrophage infiltration and oxidized lipoproteins ${ }^{[6]}$. Increased CRP causes expression of adhesion molecules and plasminogen activator inhibitor- 1 and diminishes nitric oxide production, resulting in vasoconstriction, prothrombotic, and pro-inflammatory statuses linked to endothelial dysfunction ${ }^{[7,8]}$. In the course of chronic inflammation, valvular degeneration and stenosis can be seen and its progression is also related with the inflammatory status. Valvular stenosis occurs more rapidly in case of increased inflammation. Thus, higher level of CRP causes valvular aortic degeneration and makes it stenosed. Additionally, serum CRP level is found to be correlated with valvular expression. It means that increased serum CRP levels are related with progression, severity, and prognosis of aortic stenosis. Skowasch et al. ${ }^{\left[{ }^{[]}\right.}$demonstrated that CRP localized in the aortic valve tissue of patients with aortic stenosis is correlated with serum CRP levels and it was revealed that serum CRP level is associated with progression of aortic stenosis and long-term survival in patients with asymptomatic aortic stenosis ${ }^{[3]}$. As for albumin, it is also produced in the liver and it has an antioxidant activity ${ }^{[10]}$. Contrary to CRP, albumin is reduced in the chronic inflammatory process, as a negative acute phase response protein by inflammatory substances, including IL-6 $6^{[4]}$. The decrease in the serum albumin level results in the increase blood viscosity and disruption in endothelial functions ${ }^{[11]}$.

Acute phase reactants' responses do not have to be in a similar degree for each inflammatory status. Different inflammation-based prognostic scores were identified to provide more stable regulation due to this reason. CAR, as a single inflammatory index, provides the stability against the CRP and albumin alone. In previous studies, CAR had better valuable prognostic factor to reflect inflammation status in different inflammatory diseases. Fairclough et al. ${ }^{[12]}$ demonstrated that CAR had a favorable prognostic value in elderly patients in acute exacerbations of chronic disease and that CAR was a better marker than CRP alone to predict long-term mortality in patients at intensive care units ${ }^{[13]}$. Additionally, similar results have been demonstrated in patients with malignancies, vasculitis, and critically ill patients ${ }^{[14-16]}$. CAR was also studied in cardiovascular diseases, especially in atherosclerotic coronary artery disease. Coronary artery disease severity was found to be associated with higher CAR values in patients with stable coronary artery disease and acute coronary syndrome ${ }^{[17,18]}$. This could be explained by the higher inflammatory status linked to extend of the coronary atherosclerosis. Furthermore, it was demonstrated that increased
CAR predicted poor prognosis in patients with ST-segment elevation myocardial infarction ${ }^{[19]}$.

In the light of foregoing data, increased inflammation makes the cardiac endothelium critically ill and it affects the valvular tissue, e.g., coronary atherosclerosis. Thus, cardiac valvular degeneration can be seen more distinctly, and its progression and prognosis can be worse. Unsurprisingly, in our study, it was demonstrated that higher inflammatory status was associated with poor prognosis. It is known that adverse clinical events are seen more explicitly in case of chronic inflammation ${ }^{[12-16]}$, so that higher inflammation as the underlying mechanism of the chronic degenerative valvular disease causes increased mortality rates even if the valvular disease is treated. In previous studies, increased CRP value was found to be related with poor prognosis in patients with severe aortic stenosis ${ }^{[3]}$. However, to the best of our knowledge, our study was the first to demonstrate that higher inflammatory status affected long-term mortality in patients with severe degenerative aortic stenosis undergoing surgical AVR. It could be explained by some possible underlying mechanisms. Firstly, left ventricular myocardium is affected by inflammation. Although surgical treatment of aortic stenosis is performed successfully, sufficient and completely remodeling on left ventricle cannot be seen. Thus, large inflammatory burden causes impaired left ventricular function, resulting in heart failure. It was supported in previous studies in which inflammation was found to be related with heart failure ${ }^{[20]}$. In conclusion, increased hospitalization and mortality rates can be seen due to irreversible heart failure. Supporting this, in our study, hospitalization rates after surgical AVR were found to be increased in patients with higher CAR. Secondly, inflammatory status also predicts surgical success rates. In these patients, surgical suture and prosthetic valve can become degenerative due to increased inflammatory burden. Thus, postoperative complications such as paravalvular leakage and pannus formation can affect adverse cardiac events in patients with higher inflammatory status. We also found out that paravalvular leakage ratio was higher in patients with increased CAR. While it can also be related with surgical suture technique, in this single-center population, this reason cannot affect our outcomes. Consequently, the mentioned higher CAR linked to failure of surgical treatment can result in increased aortic reoperation rates. Additionally, inflammation linked to increased risk of arrhythmia could be the other possible underlying reason of adverse clinical outcomes in this study population. Unfortunately, in our study, clinical arrhythmic events were not evaluated.

Left ventricular EF is of importance to predict adverse cardiac events and mortality in patients with aortic stenosis. It is also a determinant factor for treatment time and modality. After surgical replacement therapy, higher mortality rates may be seen in patients with left ventricular systolic dysfunction. Taniguchi et al. ${ }^{[21]}$ revealed that impaired EF was related with increased mortality rates in 3815 patients with severe aortic stenosis. Supporting these, lower EF was an independent predictor of mortality in our study. Age is another risk factor for poor prognosis in patients with valvular heart disease. Hussain et al. ${ }^{[22]}$ demonstrated that age was a predictor of mortality in 
patients with severe asymptomatic aortic stenosis undergoing surgical AVR. Also, it is known that mechanical prosthetic valves are more durable than bioprosthetic valves. Thus, bioprosthetic valves are commonly preferred in the ageing population. In our study, older age and bioprosthetic valves were found to be independent predictors of mortality due to the mentioned reasons. Additionally, ACEI or ARB usage causes decreased adverse cardiac outcomes, such as mortality, in patients with cardiovascular disease. In a meta-analysis, it was shown that treatment with ACEI or ARB was better than placebo in reducing all-cause mortality, especially in patients with chronic heart failure with reduced $\mathrm{EF}^{[23]}$. It was supported in our study by lower $A C E I$ or ARB usage ratio in patients with mortality after surgical AVR therapy.

In the light of the mentioned data, CAR is an easy, cheap, and rapid prognostic risk score to detect high-risk patients for surgical AVR. However, large scaled studies are needed to evaluate the impact of CAR on adverse clinical events in patients with severe aortic stenosis after surgical AVR.

\section{Limitations}

The major limitation of this study was a relatively small sample size to evaluate the long-term mortality. Lack of other major adverse cardiac events, such as progression of arrhythmia and cerebrovascular disease, was the other limitation. Unknown etiology of mortality in the study group due to retrospectively follow-up visits was also another crucial limitation.

\section{CONCLUSION}

Inflammatory process is known as a riskfactor of cardiovascular diseases. However, in our study, it was firstly described that higher CAR was strongly related with increased mortality rates in patients with isolated severe degenerative aortic stenosis after surgical AVR. In addition to this, rehospitalization rates due to heart failure, risk of paravalvular leakage, and aortic reoperation rates were higher in patients with increased CAR. In conclusion, CAR is an easy, cheap, and rapid prognostic risk score to detect high-risk patients for surgical AVR.

No financial support.

No conflict of interest.

\section{REFERENCES}

1. Hughes BR, Chahoud G, Mehta JL. Aortic stenosis: is it simply a degenerative process or an active atherosclerotic process? Clin Cardiol. 2005;28(3):111-4. doi:10.1002/clc.4960280303.

2. Sánchez PL, Santos JL, Kaski JC, Cruz I, Arribas A, Villacorta E, et al. Relation of circulating C-reactive protein to progression of aortic valve stenosis. Am J Cardiol. 2006;97(1):90-3. doi:10.1016/j.amjcard.2005.07.113.

3. Imai K, Okura H, Kume T, Yamada R, Miyamoto Y, Kawamoto T, et al.

\section{Authors' roles \& responsibilities}

SK Substantial contributions to the conception or design of the work; or the acquisition, analysis, or interpretation of data for the work; drafting the work or revising it critically for important intellectual content; final approval of the version to be published

ACD Drafting the work or revising it critically for important intellectual content; agreement to be accountable for all aspects of the work in ensuring that questions related to the accuracy or integrity of any part of the work are appropriately investigated and resolved; final approval of the version to be published

GD Drafting the work or revising it critically for important intellectual content; agreement to be accountable for all aspects of the work in ensuring that questions related to the accuracy or integrity of any part of the work are appropriately investigated and resolved; final approval of the version to be published

ARD Substantial contributions to the conception or design of the work; or the acquisition, analysis, or interpretation of data for the work; drafting the work or revising it critically for important intellectual content; final approval of the version to be published

EY Substantial contributions to the conception or design of the work; or the acquisition, analysis, or interpretation of data for the work; drafting the work or revising it critically for important intellectual content; final approval of the version to be published

HZA Drafting the work or revising it critically for important intellectual content; agreement to be accountable for all aspects of the work in ensuring that questions related to the accuracy or integrity of any part of the work are appropriately investigated and resolved; final approval of the version to be published

AKK Substantial contributions to the conception or design of the work; or the acquisition, analysis, or interpretation of data for the work; drafting the work or revising it critically for important intellectual content; final approval of the version to be published

FU Substantial contributions to the conception or design of the work; or the acquisition, analysis, or interpretation of data for the work; drafting the work or revising it critically for important intellectual content; final approval of the version to be published

ME Substantial contributions to the conception or design of the work; or the acquisition, analysis, or interpretation of data for the work; drafting the work or revising it critically for important intellectual content; final approval of the version to be published
C-Reactive protein predicts severity, progression, and prognosis of asymptomatic aortic valve stenosis. Am Heart J. 2008;156(4):713-8. doi:10.1016/j.ahj.2008.04.011.

4. Levitt DG, Levitt MD. Human serum albumin homeostasis: a new look at the roles of synthesis, catabolism, renal and gastrointestinal excretion, and the clinical value of serum albumin measurements. Int J Gen Med. 2016;9:229-55. doi:10.2147/IJGM.S102819.

5. Agmon Y, Khandheria BK, Jamil Tajik A, Seward JB, Sicks JD, Fought 
AJ, et al. Inflammation, infection, and aortic valve sclerosis; insights from the Olmsted county (Minnesota) population. Atherosclerosis. 2004;174(2):337-42. doi:10.1016/j.atherosclerosis.2004.01.028.

6. Sanchez PL, Mazzone A. C-reactive protein in degenerative aortic valve stenosis. Cardiovasc Ultrasound. 2006;4:24. doi:10.1186/1476-7120-4-24.

7. Feinbloom D, Bauer KA. Assessment of hemostatic risk factors in predicting arterial thrombotic events. Arterioscler Thromb Vascu Biol. 2005;25(10):2043-53. doi:10.1161/01.ATV.0000181762.31694.da.

8. Stepanova TV, Ivanov AN, Tereshkina NE, Popyhova EB, Lagutina DD. [Markers of endothelial dysfunction: pathogenetic role and diagnostic significance]. Klin Lab Diagn. 2019;64(1):34-41. doi:10.18821/0869-20842018-63-34-41. Russian.

9. Skowasch D, Schrempf S, Preusse CJ, Likungu JA, Welz A, Lüderitz $B$, et al. Tissue resident $C$ reactive protein in degenerative aortic valves: correlation with serum $C$ reactive protein concentrations and modification by statins. Heart. 2006;92(4):495-8. doi:10.1136/ hrt.2005.069815.

10. Roche M, Rondeau P, Singh NR, Tarnus E, Bourdon E. The antioxidant properties of serum albumin. FEBS Lett. 2008;582(13):1783-7. doi:10.1016/j.febslet.2008.04.057.

11. Yu Z, Tan BK, Dainty S, Mattey DL, Davies SJ. Hypoalbuminaemia, systemic albumin leak and endothelial dysfunction in peritoneal dialysis patients. Nephrol Dial Transplant. 2012;27(12):4437-45. doi:10.1093/ndt/gfs075.

12. Fairclough E, Caims E, Hamilton J, Kelly C. Evaluation of a modified early warning system for acute medical admissions and comparison with C-reactive protein / albumin ratio as a predictor of patient outcome. Clin Med (Lond). 2009;9(1):30-3. doi:10.7861/clinmedicine.9-1-30.

13. Ranzani OT, Zampieri FG, Forte DN, Azevedo LC, Park M. C-reactive protein/albumin ratio predicts 90 -day mortality of septic patients. PLoS One. 2013;8(3):e59321. doi:10.1371/journal.pone.0059321.

14. Moon JS, Ahn SS, Park YB, Lee SK, Lee SW. C reactive protein to serum albumin ratio is an independent predictor of all-cause mortality in patients with ANCA-associated vasculitis. Yonsei Med J. 2018;59(7):86571. doi:10.3349/ymj.2018.59.7.865.

15. Park JE, Chung KS, Song JH, Kim SY, Kim EY, Jung JY, et al. The C-reactive protein/albumin ratio as a predictor of mortality in critically ill patients. J Clin Med. 2018;7(10) pii:E333. doi:10.3390/jcm7100333.

16. Kinoshita A, Onoda H, Imai N, Iwaku A, Oishi M, Tanaka K, et al. The C-reactive protein/albumin ratio, a novel inflammation-based prognostic score, predicts outcomes in patients with hepatocellular carcinoma. Ann Surg Oncol. 2015;22(3):803-10. doi:10.1245/s10434-014-4048-0.

17. Karabağ Y, Çağdaş M, Rencuzogullari I, Karakoyun S, Artac I, Ilis D, et al. Relationship between C-reactive protein/albumin ratio and coronary artery disease severity in patients with stable angina pectoris. J Clin Lab Anal. 2018;32(7):e22457. doi:10.1002/jcla.22457.

18. Çağdaş M, Rencüzoğullari I, Karakoyun S, Karabağ Y, Yesin M, Artaç I, et al. Assessment of relationship between $\mathrm{C}$-reactive protein to albumin ratio and coronary artery disease severity in patients with acute coronary syndrome. Angiology. 2019;70(4):361-8. doi:10.1177/0003319717743325.

19. Çınar T, Çağdaş M, Rencüzoğulları I, Karakoyun S, Karabağ Y, Yesin M, et al. Prognostic efficacy of C-reactive protein/albumin ratio in ST elevation myocardial infarction. Scand Cardiovasc J. 2019;53(2):83-90. doi:10.10 80/14017431.2019.1590628.

20. Villacorta H, Masetto AC, Mesquita ET. C-reactive protein: an inflammatory marker with prognostic value in patients with decompensated heart failure. Arq Bras Cardiol. 2007;88(5):585-9. doi:10.1590/s0066$782 \times 2007000500014$.

21. Taniguchi T, Morimoto T, Shiomi H, Ando K, Kanamori N, Murata K, et al. Prognostic impact of left ventricular ejection fraction in patients with severe aortic stenosis. JACC Cardiovasc Inter. 2018;11(2):145-57. doi:10.1016/j.jcin.2017.08.036.

22. Hussain Al, Auensen A, Brunborg C, Beitnes JO, Gullestad L, Pettersen KI. Age-dependent morbidity and mortality outcomes after surgical aortic valve replacement. Interact Cardiovasc Thorac Surg. 2018;27(5):650-6. doi:10.1093/icvts/ivy154.

23. Burnett H, Earley A, Voors AA, Senni M, McMurray JJ, Deschaseaux $C$, et al. Thirty years of evidence on the efficacy of drug treatments for chronic heart failure with reduced ejection fraction: a network meta-analysis. Circ Heart Fail. 2017;10(1).pii:e003529. doi:10.1161/ CIRCHEARTFAILURE.116.003529. 